\title{
USO Y DEPENDENCIA DE LAS REMESAS EXTERIOR EN LA COMUNIDAD UNISUCREÑA
}

Autores: Yaneth P. Romero Álvarez (1), Dilia marcela Estrada Fúnez (2), Jorsiris Nicol Pérez Pérez (3)

\section{RESUMEN}

L as Remesas además de haberse convertido en un rubro importante dentro de la economía de los países ya que contribuyen a reducir los niveles de pobreza siempre y cuando su uso se canalice a una forma productiva. Este trabajo busca analizar el nivel de dependencia económica de los miembros de la comunidad Unisucreña desde las remesas, a través de la caracterización de éstas como herramienta de inclusión financiera, para lo cual se tomaron datos recolectados mediante encuestas aplicadas a la comunidad Unisucreña, complementado con los diferentes informes presentados por las entidades financieras y los aportes realizados por referentes teóricos acerca de este tema. Como resultado que es a pesar de ser baja la población de la comunidad que recibe remesas, se encuentra que de éste, el 57\% las utiliza para suplir las necesidades primarias del hogar. Se debe propender, a que el uso de las remesas sea más productivos de tal forma que permita generar más renta en los hogares beneficiarios .

Palabras claves: Migración, Remesas, servicios financieros Inclusión financiera

\begin{abstract}
$\mathrm{R}$ emittances have also become an important item in the economy of countries, since they contribute to reducing poverty levels as long as their use is channeled to a productive form. This paper seeks to analyze the level of economic dependence of the members of the Unisucreña community from remittances, through the characterization of these as a financial inclusion tool, for which data collected through surveys applied to the Unisucreña community was taken, complemented with the different reports presented by the financial entities and the contributions made by theoretical referents on this topic. As a result, despite being low the population of the community that receives remittances, it is found that $57 \%$ of them use them to supply the primary needs of the household. It should be encouraged that the use of remittances be more productive in such a way as to generate more income in the beneficiary households .
\end{abstract}

Keywords: Migration, remittances, financial services, financial inclusion.

\footnotetext{
(1)Artículo resultado de Trabajo de Grado derivado del proyecto de investigación "Educación financiera a través de laboratorios de aprendizaje a las comunidades de impacto de la Universidad de Sucre” aprobado mediante Resolución № 603 de 2017. Ingeniera Industrial, Magister en Finanzas, Doctorante en Sostenibilidad de la Universidad Politécnica de Catalunya y docente-Investigador Universidad de Sucre; Correo electrónico: yaneth.romero@unisucre.edu.co (2) Estudiante Administración de Empresas, Universidad de Sucre. Correo electrónico: dilia-marce@outlook.es. (3) Estudiante Administración de Empresas, Universidad de Sucre. Correo electrónico: jor0679@hotmail.com
} 


\section{USO Y DEPENDENCIA DE LAS REMESAS EXTERIOR EN LA COMUNIDAD UNISUCREÑA}

\section{INTRODUCCIÓN}

$\mathrm{E}^{\prime}$ ser humano está en constante lucha con el medio que lo rodea para poder vivir en condiciones cada vez mejores, es por ello, que desde hace muchos años las migraciones a nivel mundial han aumentado, debido a la búsqueda incesante de una mejor calidad de vida que otros países pueden ofrecer a estos ciudadanos llegando a convertirse en un fenómeno social y económico que abarca a todo el mundo. Todo esto ha ocasionado que los países involucrados en estos procesos lo tomen como un gran reto que deben afrontar, puesto que existe una preocupación por el nivel de dependencia que éstas puedan ocasionar en los hogares beneficiarios, con respecto al uso que se les da y la poca participación de estas familias en el sistema financiero.

Durante mucho tiempo las remesas pasaban por desapercibidas, no se les daba la mayor importancia a estos flujos de dinero, "debido a que la gran mayoría de las personas que envían remesas y las familias que las reciben no recurren a bancos" (BID, 2001), pero este concepto ha ido evolucionando, tanto así que las remesas conforman un rubro importante en la estabilidad económica de un país. Tal es el caso de Colombia, en el que se ha visto esto reflejado en su balanza de pagos; ocasionando que muchos hogares dependan de ellas para subsistir y propender a un mejor bienestar. Ante esta situación, el gobierno se ha visto en la necesidad de buscar estrategias que posibiliten la inclusión financiera desde las remesas, pero hasta el momento ha sido un proceso difícil.

La Costa Caribe, no ha sido ajena a este proceso, puesto que el porcentaje de participación es de un $7.6 \%$ de las remesas que ingresan al país y al departamento de Sucre, llega el $0.3 \%$ del total de ingresos que se recibe desde el exterior (Banco de la República, 2014) y aunque se considera un porcentaje bajo, las remesas aun así, se convierten en un esta- bilizador económico de muchas familias, ya que su uso está destinado a satisfacer necesidades básicas aunque no generen un aumento de capital para sus beneficiarios y además no mejoran la competitividad del país ante el mundo.

En este sentido el objetivo general de esta investigación es analizar el nivel de dependencia económica de los miembros de la comunidad Unisucreña desde las remesas, a través de la caracterización de éstas como herramienta de inclusión financiera.

\section{ANTECEDENTES}

$\checkmark$ on numerosas las investigaciones encontradas en cuanto al estudio de la dependencia y uso de las remesas. A nivel internacional podemos encontrar el de Reyes y Gijon (2005), que buscó desmitificar a las remesas como fuente de financiamiento del desarrollo local en comunidades rurales del estado de Oaxaca en México, midiendo los niveles de dependencia económica de las remesas, concluyendo que la falta de instituciones que ofrecen servicios micro-financieros en las localidades rurales no permite que las remesas se reciclen y se fuguen a los centros regionales de mercado. Cerrutti (2008) por su parte realizó un estudio similar cuyo propósito era establecer las diferencias en los patrones de envío y la utilización de remesas de mujeres y varones en el caso de dos comunidades migratorias en Argentina que presentan rasgos diferenciados, la de Paraguayos y Peruanos y de esta manera identificar los factores asociados al envío de remesas, monto, frecuencia y utilización de estas en el lugar de destino. 


\section{USO Y DEPENDENCIA DE LAS REMESAS EXTERIOR EN LA COMUNIDAD UNISUCREÑA}

En este estudio, los resultados determinaron que la situación familiar tanto en el país de origen como en el de destino de los migrantes tiene una fuerte influencia en la probabilidad de remitir, y los métodos de envío cuando se trata de montos pequeños, utilizan parientes o conocidos que viajan a Paraguay, mientras que los peruanos las remitieron utilizando agencias de envío de dinero, este dinero es utilizado tanto en las familias peruanas como paraguayas para solventar los gastos de mantenimiento de los hogares.

También se resalta el trabajo de Castro (2004), que tenía por objetivo determinar el impacto de las remesas en el nivel de bienestar de los hogares salvadoreños, así como la proporción que éstas representan en los ingresos de hogares, las diferencias socioeconómicas entre los hogares receptores y los que no obtienen ingresos de este tipo, comparando además, la situación de pobreza de los salvadoreños con el contexto hipotético de no recibir remesa alguna; el hallazgo principal de dicha investigación fue que si las remesas no existieran o si nunca se hubiera presentado el fenómeno migratorio estudiado, habría aumentado la pobreza o surgido la miseria en esos hogares pobres, ahondando que la causa principal de la migración es la búsqueda de mejores condiciones de vida.

A nivel nacional, en Colombia, se destaca el estudio realizado por Roa (2010), cuyo objetivo era describir los usos de las remesas y explorar algunas causas que llevan a los hogares en Cali, con experiencia migratoria en España; encontrándose que, sin el aporte de las remesas, casi todos los hogares encuestados estaban casi que, condenados a la pobreza, debido a sus bajos niveles de ingresos y a la ausencia de movilidad social. Entre uno de los estudios más amplios llevado en el país, se encuentra el de "Emigración Internacional en el Área Metropolitana Centro Occidente Colombia AMCO", un cuadernillo que hace parte de una serie de investigaciones referentes a este tema, en el cual se llega conclusiones importantes sobre el tema; las remesas se han convertido en una forma de ingresos "cuasipermanente", aumentando la probabilidad de que éstas sean incorporadas en los hábitos de consumo y gasto de los hogares, por otro lado, la investigación resalta que de los hogares receptores de estos recursos, destinan tan solo el 6,4\% de los gastos de los negocios que poseen los hogares beneficiaros, son financiados con estos recursos; es de resaltar que las encuestas fueron realizadas únicamente a beneficiarios de las remesas de trabajadores en las casas de cambio (Garay y Rodriguez, 2005).

\section{MARCO TEÓRICO}

\section{El fenómeno de la migración}

S e conoce como migración a desplazamientos $\mathcal{D}_{\text {de la población desde su lugar de origen a un }}$ lugar de destino diferente al de su procedencia en busca de mejorar sus condiciones de vida, originados por problemas económicos, culturales, sociales etc. (OIM, 2006). Un fenómeno migratorio es una movilidad geográfica de personas, de manera individual o en grupo que se desplazan a hábitats distintos al de su cotidianeidad (Forero, 1993), movilidad, que no es en un tiempo determinado y que seguramente encontrará las dificultades crecientes de la libre circulación de personas que acompaña a la globalización actual, como lo afirman Zuñiga, Gil, Atienza, Malgesini, Niberg Hear y Herrera (2005). La migración se da a nivel mundial, por ello, la circulación de personas alrededor del mundo, afecta a los países sea de forma positiva o negativa, dependiendo de las políticas públicas de cada país para asumir la entrada y salida de personas, todo este proceso genera el aumento de remesas, las cuales 


\section{USO Y DEPENDENCIA DE LAS REMESAS EXTERIOR EN LA COMUNIDAD UNISUCREÑA}

pueden contribuir a mitigar la pobreza o propiciar el desarrollo.

Debido a los diferentes tipos de migración que existen y las diversas causas que han surgido con el pasar del tiempo, resulta difícil obtener datos exactos acerca de del total de los migrantes que entran o salen de un determinado territorio, por lo que se ha recurrido a la realización de censos por parte del gobierno, lo que da un acercamiento a esas cifras. De acuerdo a Zuñiga, et al. (2005), las migraciones no se establecen o se originan al azar, por el contrario, se relacionan estrechamente con acontecimientos históricos, económicos, sociales y políticos que marcan a la sociedad, además también por el incremento de las necesidades de los seres humanos, la violencia y hasta la misma pobreza, por falta de desarrollo de su país origen. A pesar de que la migración se constituye como una solución a los problemas que afrontan las personas con bajo poder adquisitivo o para aquellas personas que buscan un mejor futuro, se vuelve un factor preocupante cuando ésta se presenta en economías pequeñas, puesto que se da el flujo de capital intelectual hacia otros países ya desarrollados. La emigración de profesionales de las distintas áreas del conocimiento se considera una pérdida para el país de origen, pero a la vez representa aumento de la competitividad en los países de destino. De acuerdo a lo anterior, el consenso entre los economistas es que la inmigración favorece el crecimiento económico, pues no solo mejora la vida de los inmigrantes sino también la de quienes ya viven en el lugar (Appelbaum, 2017).

La migración en Colombia es establecida por Gaviria ( citado en Roa, 2011), por la difícil situación económica por la inestabilidad y falta de empleo (precariedad laboral), la inseguridad y el conflicto armado en el país, la influencia de familiar que facilitó la migración, y la mejor calidad de vida en otros países. Cabe destacar que otra de las razones que originan la migración de personas colombianas a otros países, en especial las mujeres, es su espíritu materno, pues están dispuestas a todo tipo de sacri- ficios con tal de darles una mejor calidad de vida a sus hijos y de sacarlos adelante (Rivas y Gonzálvez, 2011). Se considera que existen 2,5 millones de colombianos emigrantes aproximadamente en todo el mundo (The World Bank, 2016), más aún, según Migración Colombia (2017), en su boletín migratorio para diciembre de 2017 la cantidad de viajeros fue de 1.455.481 que pertenece al ingreso y salida del país de nacionales y extranjeros, del total, el $56 \%$ muestra los movimiento de los nacionales y el $44 \%$ restante corresponde al flujo de viajeros extranjeros. En cuanto a los colombianos que salieron al exterior la cifra es de 397.780, que corresponde al $49 \%$ del total de movimiento de colombianos.

Con respecto a la emigración de los colombianos al exterior, los países de destino más frecuentes son Estados Unidos, España, Chile y Canadá (Pérez, 2013). Específicamente en el caso de Colombia las migraciones están altamente concentradas en pocas regiones expulsoras y pocos países receptores $\mathrm{La}$ mayoría de los migrantes que viven en Estados Unidos y España vienen del Eje Cafetero y Valle del Cauca y, en menor medida, de Antioquia y Bogotá (Bonilla, 2016).

\section{Remesas}

Esta ola de migraciones dada en el mundo ha traído consigo el aumento de las remesas, las cuales se pueden definir según Meyers (2000), como los ingresos monetarios que ganan los emigrantes trabajando en el extranjero y luego envían a su país natal, es decir, las remesas se convierten en una consecuencia activa de la migración internacional, en la que los movimientos incesantes de personas en busca de trabajo en otros países generan flujos de 


\section{USO Y DEPENDENCIA DE LAS REMESAS EXTERIOR EN LA COMUNIDAD UNISUCREÑA}

dinero a sus países de origen, convirtiéndose éstos en una fuente de ingresos para las familias de los migrantes, como un medio para mantener los lazos afectivos y de dependencia económica, así como una muestra del compromiso afectivo y emocional que mantiene con sus familiares (Rivas y Gonzálvez, 2011). Los remitentes de remesas se caracterizan por emigrar en una edad laboral productiva, con el fin de alcanzar sus metas personales o profesionales en el exterior (López, Díaz, Sierra, Tabera, y Contreras, 2008), por lo tanto, se configuran como migrantes laborales, es decir, una población económicamente activa.

\section{Usos de las Remesas}

Las remesas juegan un papel importante en el desarrollo de los países, ya que además de incrementar el ingreso de éstos, contribuyen a reducir los niveles de pobreza y a mejorar la distribución de la riqueza en las familias (Hernández y Silva, 2017). Los usos de las remesas se han ido diversificando con el pasar del tiempo, al igual que el aumento de las necesidades, gustos y prioridades de las personas, y dependiendo del uso que se les dé, se pueden clasificar como de ahorro, inversión y capital. Autores como Chami, Fullenkamp y Jahjah (citados por Gómez, 2016) hacen referencia a que gran parte de las remesas que envían los emigrantes a los países de destino se gastan principalmente en bienes de consumo; y solo una parte pequeña se destina a inversión o ahorro; en el caso de Colombia, según el estudio de remesas internacionales del año 2012, realizado en marzo de este mismo año por el Fondo Multilateral de Inversiones, el Centro de Estudios Monetarios Latinoamericanos y el Banco Interamericano de Desarrollo el principal uso que se le da a las remesas corresponde a vivienda con un 15,5\% (incluye arrendamiento y adquisición de vivienda), servicios públicos con un $23,30 \%$ y alimentos un 29,3\% (BID, 2018).

Según Withers (2017) el envío de remesas aumenta el consumo del hogar y en ocasiones se convierten en el principal ingreso de las familias receptoras, además de ser utilizadas para el pago de deudas, manteniéndose por encima de la línea de pobreza (Barb, 2008). Sólo para el año 2015, el valor de las remesas enviadas por los colombianos al Eje Cafetero fue aproximadamente de 278,6 millones de dólares, de los cuales el 15,5\% se utilizó para pagar el canon de arrendamiento y las cuotas de los préstamos para adquisición de vivienda, (Salazar, 2017) . Generalmente el dinero que se envía, es utilizado para satisfacer las necesidades básicas de cada uno de sus miembros, luego de esto, buscan invertirlas en otras cosas como: compra de artículos para el hogar, ropa, entre otros, posteriormente cuando ya se ha cubierto lo primordial deciden invertirlo en pequeños negocios, adquisición de propiedades, etc. En base a esto, se puede decir que las remesas por ser destinadas en su mayor parte para la compra de bienes y pago de servicios; inciden positivamente en la economía, ya que incentivan el consumo, lo que conlleva a que se les considere parte importante del crecimiento económico de los países en desarro1lo. Incluso, aún más importante, autores como Tedesco (citado por Pinzón y Silva, 2016), aseguran que en América Latina el ingreso de dinero por concepto de remesas ha permitido mejorar las condiciones de vida de los hogares receptores y ha contribuido en la disminución de la mortalidad infantil.

Sin embargo, el envío de remesas a familiares produce una nueva dificultad. De acuerdo a (Pinzón y Silva, 2016) existen casos en los cuales las remesas se convierten directamente en el principal ingreso para financiar las necesidades básicas de las personas que se encuentran en el mercado laboral, como es el caso de los desempleados a los que este dinero les permite solventarse económicamente durante 


\section{USO Y DEPENDENCIA DE LAS REMESAS EXTERIOR EN LA COMUNIDAD UNISUCREÑA}

el lapso de tiempo en el que buscan empleo, lo que conlleva a disminuirla la probabilidad de que estas personas se inserten de nuevo al mercado laboral (Mora, 2008).

En el contexto internacional, en países como México, Nicaragua, Guatemala y Ecuador y Paraguay, el uso dado a las remesas, es para financiar el gasto corriente de las familias (gastos de primera necesidad de la familia como ropa, alimentos, servicios públicos, transporte y medicinas), le siguen los gastos en educación y salud Altamirano, Castillo y Rodríguez, 2015; Cachago y Mafla, 2016; Gaibor, García, y Songor, 2017; Gómez, 2016; López, 2017; Pardo y Dávila, 2017; Uribe, 2005). Otro aspecto estudiado, además del uso de las remesas, son los montos que se envían por concepto de remesas. A nivel internacional, se observa que para algunos continentes las remesas tienen grandes aumentos, mientras que para otros disminuye, esto depende de la estructura interna de cada país. Es así como se estima que las remesas a los países en desarrollo llegaron a 429,000 millones en el 2016, un declive de $2.4 \%$ sobre el 2015 . De manera similar, las remesas globales, que incluyen envíos a países ricos, cayeron $1.2 \%$ a 575,000 millones. La India mantuvo su posición como el mayor receptor mundial de remesas, pero también lideró la caída con un monto de 62,000 millones de dólares, un agudo retroceso de $8.9 \%$ respecto al 2015." (AFP, 2017)

Entre los principales receptores de remesas registradas oficialmente por el Banco Mundial en el año 2014 se encuentran: India (US\$70 000 millones), China (US\$64 000 millones), Filipinas (US\$28 000 millones) y México (US\$25 000 millones). Otros grandes destinatarios son: Nigeria, Egipto, Pakistán, Bangladesh, Viet Nam y el Líbano. Sin embargo, como proporción del producto interno bruto (PIB), las remesas fueron más cuantiosas en países más pequeños y de ingreso más bajo; los mayores receptores en relación con el PIB en 2013 fueron: Tayikistán (49\%), República Kirguisa (32\%), Nepal
(29\%), Moldova (25\%), Tonga (24\%), y Haití y Armenia (ambos con 21 \%)." (Banco Mundial, s.f). El continente europeo, más que recibir remesas se caracteriza por ser uno de los destinos preferidos de los migrantes, por su alto grado de desarrollo y la necesidad de mano de obra. "Los inmigrantes en Europa enviaron 109.400 millones de dólares (unos 97.500 millones de euros) en 2014 a sus familias, 1o que supone un $25 \%$ de las remesas mundiales, informó hoy en Roma el Fondo Internacional de Desarrollo Agrícola (FIDA)." (EFE, 2015), "los países donde se generaron más remesas fueron, según el estudio, Rusia, el Reino Unido, Alemania, Francia, Italia y España" (Centro de Noticias ONU, 2015), el dinero que sale de Europa para los países en vía de desarrollo, es destinado principalmente para suplir las necesidades primarias. Por otro lado, en el continente asiático, las remesas hacia Asia meridional crecieron un $2 \% \%$ en 2015 , una baja con respecto al 4,3\% de 2014." (Banco Mundial, 2016). Es de hacer notar, que uno de los países que más envían remesas a nivel del mundo, se encuentra Arabia Saudita y Emiratos Árabes, pero a su vez, llega una gran cantidad de extranjeros, que se quedan a laborar y giran dinero a sus países natales, donde vive su familia. China siendo una potencia a nivel mundial, es un país, que capta una buena cantidad de dinero por conceptos de remesas, ya muchos habitantes de este país se encuentran laborando en otros países. (Murillo, 2017).

Las remesas en América latina y el Caribe, alcanzaron un récord en el año 2016, "Los principales aspectos que caracterizaron la evolución de ese ingreso fueron los siguientes: i) las remesas incrementaron a 70,369 millones de dólares, con lo que sumaron aumentos durante siete años consecutivos; 


\section{USO Y DEPENDENCIA DE LAS REMESAS EXTERIOR EN LA COMUNIDAD UNISUCREÑA}

ii) el crecimiento anual de ese ingreso del exterior resultó del 7.2\%, lo que representó la tasa más alta registrada en los últimos 10 años; iii) el incremento de las remesas fue generalizado en las distintas subregiones; y iv) se originó, entre otros factores, por aumentos en los niveles de empleo, de remuneraciones medias y consecuentemente de la masa salarial de los trabajadores emigrantes de la región en los principales países de destino". (Maldonado, Cervantes, \& Bonilla, 2017) establecieron en su estudio que un total de 23 millones de hogares en todo el subcontinente reciben remesas, con 16,6 millones de remesas procedentes de Estados Unidos" (EFEUSA, 2017), por otro, lado Paul Bances, citado por (EFEUSA, 2017), " director de la firma de envío de dinero MoneyGram para Latinoamérica, señaló que la subida de la cuantía de las remesas se debe también al aumento de las destrezas profesionales de los nuevos inmigrantes, lo que les permite enviar más dinero a sus familias."

En el contexto nacional, al revisar la balanza de pagos en Colombia, se encuentra que "en términos de los ingresos corrientes de la balanza de pagos, en 2016, los ingresos por remesas de trabajadores ascendieron a US\$ 4.858 con un incremento anual del $4,8 \%$. Estos ingresos son equivalentes a $1.7 \% \mathrm{del}$ PIB y a $9 \%$ de los ingresos corrientes de la balanza de pagos. Por país de origen, los incrementos más importantes se observaron en las remesas enviadas desde Estados Unidos y América Latina, destacándose el aumento de las originadas en Chile, Ecuador y Panamá. En conjunto, las remesas que se originan en Estados Unidos y España representaron el $64 \%$ del total recibido en 2016. Los factores que explican este crecimiento son principalmente la recuperación de la producción y del empleo en estos países" (Banco de la República, 2017); Por lo tanto, en Colombia los ingresos que entran por modalidad de remesas se concentran principalmente en el departamento del Valle del Cauca, con una participación del 29,4\% para el año 2014 y seguido de este se encuentran los departamentos de Antioquia, Cundinamarca y Risaralda.

En cuanto a los medios para enviar las remesas, existen diversos canales que son usados por los migrantes para enviar el dinero a su familia, formales e informales, esto depende en gran medida de los menores costos que ofrezcan los diversos medios para su elección. Khoudour (2007), resume en la Figura 1, los medios para enviar las remesas, en una pirámide que la denomina tipología de las remesas

Figura 1: Tipología de las remesas.

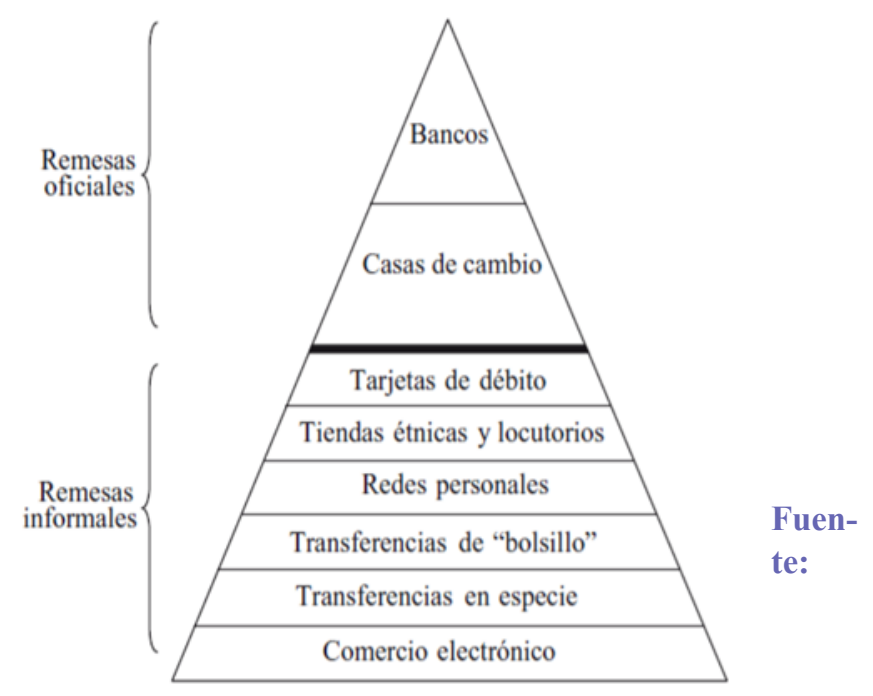

(Khoudour, 2007)

Por esta diversidad de medios de envío, se hace difícil cuantificarlas, por lo que en ocasiones cuesta analizar de forma concluyente los efectos positivos o negativos de las remesas en una economía. En Colombia, Asobancaria (2016) ha determinado tres medios para el envío del dinero desde el extranjero, éstos son: 


\section{USO Y DEPENDENCIA DE LAS REMESAS EXTERIOR EN LA COMUNIDAD UNISUCREÑA}

Bancos: Puede enviar dinero a una cuenta en Colombia directamente desde su cuenta bancaria creada en el exterior, utilizando como método de envío banco a banco.

Compañías de transferencia de dinero: se entrega el dinero que se va a transferir en una oficina y en poco tiempo el dinero ya estará en Colombia, es medio es efectivo a efectivo, la forma de enviarlas.

Plataformas online: con estas plataformas el envío se hace desde la casa o cualquier lugar donde se encuentre el migrante con acceso a internet mediante transferencias electrónicas entre bancos asociados, para que sean retiradas por ventanilla o abonadas en una cuenta de ahorros o corriente en Colombia, implementando el internet como herramienta para proceder a realizar el envío.

\section{METODOLOGÍA}

$\mathrm{L}$ a investigación planteada, es de naturaleza, Lexploratoria, descriptiva y transversal, con un enfoque cuantitativo ya que usa la recolección de datos para probar hipótesis, con base en la medición numérica y el análisis estadístico, para establecer patrones de comportamiento y probar teorías (Hernández, Fernández y Baptista, 2006). La investigación se llevó a cabo en la Universidad De Sucre, perteneciente a la ciudad de Sincelejo en Colombia. Se aplicó como herramienta de recolección de información la encuesta, realizada a estudiantes, docentes administrativos y servicios generales, de las distintas sedes de la Universidad. La información fue recopilada por medio de fuentes primarias y secundarias.

El tamaño de la muestra seleccionada para cada tipo de encuestado se calculó con la fórmula del tamaño cuando se conoce el tamaño de la población (1) y sus resultados para un nivel de confianza del 95\%, una probabilidad igual de éxito o fracaso y un margen de error del 5\% se muestran en la Tabla 1.

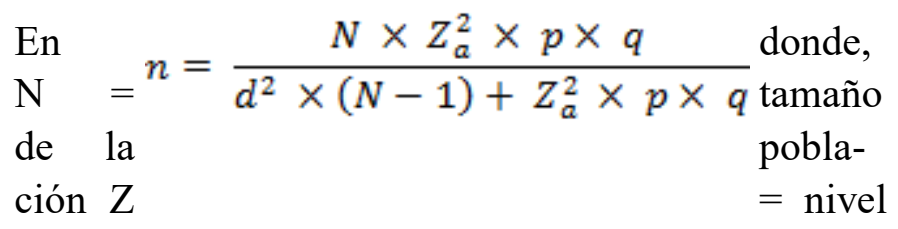
de confianza, $\mathrm{P}=$ probabilidad de éxito, o proporción esperada $\mathrm{Q}=$ probabilidad de fracaso $\mathrm{D}=$ precisión (Error máximo admisible en términos de proporción).

Tabla 1: Tamaño de las muestras poblacionales

Fuente: Elaboración propia.

\begin{tabular}{lc} 
Tipo de comunidad & $\begin{array}{c}\text { Mínimo de en- } \\
\text { cuestas }\end{array}$ \\
\hline Estudiantes & 362 \\
\hline Docentes & 188 \\
\hline Administrativos & 132 \\
\hline Servicios varios & 28 \\
&
\end{tabular}

A partir de la información recolectada a través del cuestionario cerrado, en la muestra seleccionada, se encuentra que, del total de la muestra poblacional de la comunidad de la Universidad de Sucre, sólo el $8 \%$ (Figura 2) son beneficiarias de las remesas del exterior Excluyendo a la población que afirma que no reciben remesas, la muestra de 42 personas se caracteriza porque $45 \%$ se encuentra en un rango de edad 16-26 años, lo que indica que en su mayoría son los estudiantes los mayores beneficiarios de remesas, el $10 \%$ que están entre los 27 y 37 años, el $29 \%$ tienen 38 a 48 y el $17 \%$ se encuentra entre los 


\section{USO Y DEPENDENCIA DE LAS REMESAS EXTERIOR EN LA COMUNIDAD UNISUCREÑA}

49 y 59 años. Por la variable sexo, el $50 \%$ de los que afirman recibir remesas son mujeres y el otro $50 \%$ son hombres.

Figura 2: Población beneficiaria de Remesas

\section{Beneficiarios de Remesas de la Comunidad Unisucreña}

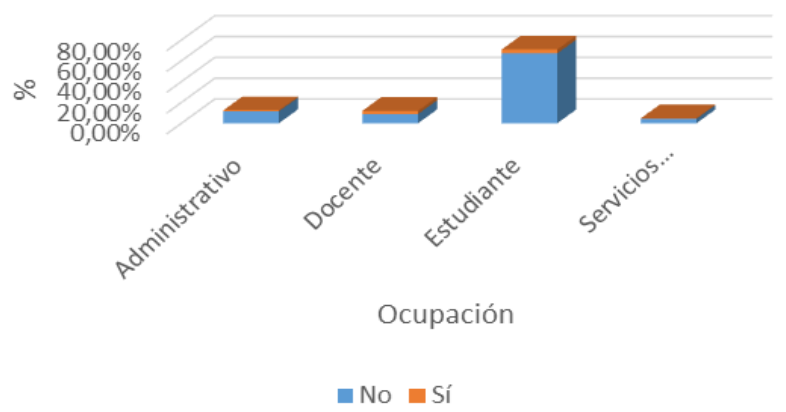

Fuente: Elaboración propia

Los medios más utilizados para recibir las remesas en la comunidad Unisucreña de acuerda a la Figura 3 , es a través de los bancos con $48 \%$, seguido de un $45 \%$ que las reciben a través de las empresas de giro como la Western Union y finalmente el 7\% las recibe con un tercero que viaja. Llama la atención que la sumatoria de los medios por el cual son recibidos estos ingresos (empresas de giro y con un tercero) superan al porcentaje que las recibe por medio de los bancos.

Figura 3: Porcentaje por medio utilizado para recibir las remesas en la comunidad de la Universidad de Sucre.

\section{Medios para recibir las remesas comunidad} Unisucreña

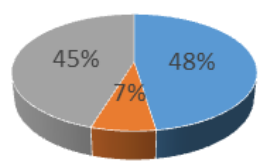

- Bancos

- Con un tercero que viaja

- Empresas de giros como Western Union

Fuente:
En cuanto a los montos, el $43 \%$ recibe entre $\$ 100.000$ y $\$ 700.000$, seguido del $36 \%$ que recibe $\$ 700.000$ a $\$ 1.000 .000$, el $16 \%$ recibe más de $\$ 1.000 .000$ y tan solo el 5\% recibe menos de $\$ 100.000$

Por último, la variable para la investigación que determina el nivel de dependencia económica de los miembros de la comunidad Unisucreña, es el uso que los miembros de la Universidad de Sucre les dan a estos recursos, de acuerdo a la figura 9, el $57 \%$ las utiliza para los gastos mensuales de la casa, un $19 \%$ para pagar algún otro tipo de crédito, otro $19 \%$ para otros usos, el 3\% para gastos personales y de educación y tan solo un $2 \%$ las destina para inversión y poder generar más capital.

Figura 4: Porcentaje del uso dado a las remesas por la comunidad de la Universidad de Sucre .

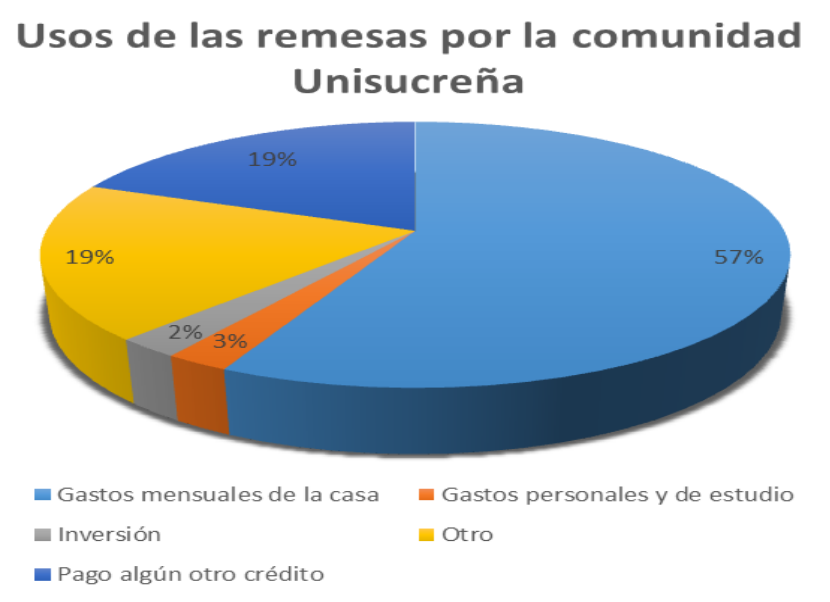

Fuente: Elaboración propia

Sobre su país de procedencia, el 33\% de las remesas que reciben los miembros de la Universidad provienen de Estados Unidos, le sigue Panamá con un $17 \%$, le sigue Ecuador y Venezuela con un $10 \%$. Pero lo que llama la atención es que un $2 \%$ de los beneficiarios de las remesas afirma que este dinero es proveniente de Colombia, esto da a entender que no tiene conocimiento claro del concepto de remesas. 


\section{USO Y DEPENDENCIA DE LAS REMESAS EXTERIOR EN LA COMUNIDAD UNISUCREÑA}

\section{CONCLUSIONES}

$\mathrm{C}$ on el paso del tiempo las remesas se han convertido en un rubro importante dentro de la economía del país, debido al auge que ha tomado este producto financiero en los últimos tiempos, sin embargo el difícil acceso al sistema financiero ha ocasionado que las personas le hagan poco uso de estos canales formales para el envío de estas; además de la utilización poco productiva que le han dado los receptores ha originado que no se aprovechen otros beneficios que estas tienen, frenando así el crecimiento y desarrollo que le pueden generar al país. Una de las principales razones que ha existes para que las personas no reciban las remesas por medio del sistema financiero, es por la falta de información hacia este, así como la falta de acompañamiento de las entidades financieras a estas y el escaso diseño de productos y servicios de acuerdo a las necesidades de ellos.

Para combatir este problema organizaciones como el Banco mundial han implementado programas de educación financiera que puedan promover el uso eficiente de productos financieros para que las personas lo conviertan en capital productivo, en Colombia el Gobierno nacional en compañía de los establecimientos financieros han diseñado una estrategia de educación financiera que le permitirá a las personas de bajos ingresos acceder a productos financieros que satisfagan sus necesidades y que ayuden a mejorar su calidad de vida.

Esta investigación establece el nivel de dependencia económica de los miembros de la comunidad Unisucreña producto financiero las remesas, obteniendo como resultado que el $57 \%$ de los beneficiarios las utiliza para suplir las necesidades primarias del hogar, es decir son usadas en los gastos mensuales de la casa que permiten mejorar su calidad de vida, que aumentan el consumismo y que de una manera $\mathrm{u}$ otra apoyan al crecimiento de la economía pero que poco impactan en el desarrollo sostenible de esta, ya que no están creando empleos o generando inversiones que permitan aumentar las ganancias a estos flujos de dinero y reduzcan la dependencia económica de las familias a estas.

Este establecimiento del nivel de dependencia, permite analizar que, aunque del total de la población encuestada, el $8 \%$ son beneficiarios de remesas y que de este porcentaje su principal uso son los gastos de la casa, el monto recibido por el $43 \%$ de esta población está entre los $\$ 100.000$ y $\$ 700.000$ mtce Cop y que el principal país de origen es Estados Unidos, se puede decir que el diferencial cambiario beneficia más a estos en cuanto al volumen recibido. Está población es dependiente a las remesas por el uso recibido. Se debe propender, a que el uso de las remesas sea más productivos de tal forma que permita generar más renta en los hogares beneficiarios. 


\section{USO Y DEPENDENCIA DE LAS REMESAS EXTERIOR EN LA COMUNIDAD UNISUCREÑA}

\section{BIBLIOGRAFÍA}

AFP. (23 de Abril de 2017). Remesas hacia América Latina aumentaron 73,000 millones de dólares en el 2016: BM. El economista. Obtenido de https:// www.eleconomista.com.mx/sectorfinanciero/

Remesas-hacia-America-Latina-aumentaron-73000millones-de-dolares-en-el-2016-BM-20170423-

0034.html

Altamirano Vindell, J., Castillo Barreda, L., \& Rodríguez López, E. (2015). Incidencia de las remesas familiares en el nivel de vida delos hogares receptores del distrito I, de la ciudad de Estelí. Universidad Nacional Autónoma de Nicaragua Unan Managua/. Obtenido de http:// repositorio.unan.edu.ni/2001/1/17351.pdf

Appelbaum, B. (10 de Agosto de 2017). ¿Si hay menos inmigrantes habrá mas trabajo? Los economistan no lo creen. The New York Times, pág. 6.

Asobancaria. (21 de Octubre de 2016). ¿Cómo enviar remesas a Colombia? Obtenido de Asobancaria: http://www.asobancaria.com/sabermassermas/ como-enviar-remesas-colombia/

Banco de la República. (2014). Principales depertamentos receptores de remesas en Colombia. Principales resultados de la encuesta trimestral de remesas. Obtenido de http://www.banrep.gov.co/remesas

Banco de la República. (2017). Evolución de la Balanza de Pagos y Posición de Inversión Internacional. Bogota, D.C.

Banco Mundial. (13 de Abril de 2016). Banco Mundial Org. Obtenido de Banco Mundial Org: http:// www.bancomundial.org/es/news/press-

release/2016/04/13/remittances-to-developingcountries-edge-up-slightly-in-2015

Banco Mundial. (s.f). Banco Mundial org. Obtenido de Migración y remesas: panorama general: http:// www.bancomundial.org/es/topic/

migrationremittancesdiasporaissues/overview
Barb, T. (2008). Remesas, pobreza y trabajo. Estudio sobre el barrio Cuba. Pereira-Colombia, 2008. Cuadernos Migrantes N.1.

BID. (2001). Remesas de inmigrantes: moneda de cambio económico y social. Washington, D.C.

Bonilla Mejía, L. (2016). Choques Externos y Remesas Internacionales en las Regiones de colombia. Documento de trabajo sobre Economía Regional, Banco de la República, Centro de Estudios económicos Regionales (CEER)- Cartagena, 54.

Cachago Lluglluna, E. J., \& Mafla Urresta, D. A. (Mayo de 2016). Escuela Politecnica Nacional. Recuperado el 26 de abril de 2018, de Escuela Politecnica Nacional: http://bibdigital.epn.edu.ec/ handle/15000/15238

Castro, Á. (2004). El impacto de las remesas en la pobreza de ingresos de los hogares salvadoreños. Realidad: Revista de Ciencias Sociales y Humanidades, 37-66.

Centro de Noticias ONU. (2015). ONU. Obtenido de http://www.un.org/spanish/News/story.asp? NewsID=32601\#.WlwHPqjibIU

Cerrutti, M. (2008). El envío de remesas de migrantes peruanos y paraguayos en el área metropolitana de Buenos Aires: un estudiocomparativo.

EFE. (15 de Junio de 2015). EFE:Agencia EFE. Obtenido de https:/www.efe.com/efe/espana/ portada/el-25-de-las-remesas-migrantes-sale-europa -segun-un-estudio/10010-2639516

diciembre_4.pdf 


\section{USO Y DEPENDENCIA DE LAS REMESAS EXTERIOR EN LA COMUNIDAD UNISUCREÑA}

EFEUSA. (10 de Febrero de 2017). EFE: Agencia EFE. Obtenido de https://www.efe.com/efe/ america/ame-hispanos/remesas-a-latinoamericaaumentan-en-2016-medio-de-las-promesasmuros/20000034-3175914

Forero, E. S. (1993). Migración e Identidad:Experiencias del Exilio. México: Publicidad, arte y serigrafia.

Gaibor Monteros, J., García, P., \& Songor, X. (2017). Remesas isalida de la desigualdad en países latinoamericanos? Un estudio comparativo entre Ecuador y México. Revista Nuestra América, 140159.

Garay Salamanca, L., \& Rodriguez Castillo, A. (Abril de 2005). La emigración internacional en el área metropolitana centro occidente Colombia. Caracterización socioeconómica de la población emigrante y evaluación del impacto de las remesas internacionales. Bogotá, Colombia.

Gómez, P. S. (2016). Remesas internacionales sursur y norte-sur en Paraguay: patrones sociodemográficos, destino de los fondos y medios de circulación. SCIELO.

Hernández Rivera, A., \& Silva Vázquez, J. C. (2017). Análisis del flujo de remesas de 20152017:Implicaciones sociales y financieras. México.

Hernández Sampieri, R., Fernández Collado, C., \& Baptista Lucio, P. (2006). Metdología de la investigación. Cuarta edición. México: Mcgraw-Hill.

Khoudour, D. (2007). Migraciones internacionales y desarrollo: el impacto socioeconómico de las remesas en Colombia. Revista de LA CEPAL, 143161.

López, E. B. (2017). Remesas y desarrollo en Guatemala. Revista Analisis de la Realidad Nacional, 64-75.

López, M., Díaz, M., Sierra, M., Tabera, B., \& Contreras, A. (2008). Perfil socioeconómico del emigrante de Medellín a España: una aproximación a partir de la experiencia de la red. Lectura de la Economía, 85-113.

Maldonado, R., Cervantes, J., \& Bonilla, S. (2017). Las remesas hacia América Latina y el Caribe en 2016: Nuevo Récord. México. Obtenido de http:// www.cemla.org/PDF/remesaseinclusion/2017-06acelerandoremesas.pdf

Meyers, D. W. (2000). Remesas de América Latina: revisión de la literatura. Comercio Exterior, 275288.

Migración Colombia. (Diciembre de 2017). Migración Colombia. Recuperado el 09 de Marzo de 2018, de Migración Colombia: http:// migracioncolombia.gov.co/index.php/es/ component/content/article?id $=718$

Mora, J. J. (2008). La relación entre la participación laboral y las remesas en Colombia. Borradores de Economía y Finanzas - ICESI.

Murillo, J. (08 de Julio de 2017). ¿Cuáles son los países con mayores envíos de remesas? Expansión. Obtenido de http://www.expansion.com/ economia/2017/07/08/595e0d08ca47410c5a8b4624.html

OIM. (2006). Organización Internacional para las Migraciones. Obtenido de Organización Internacional para las Migraciones.

Pardo Montaño, A., \& Dávila Cervantes, C. (2017). Migración y desarrollo. Características de los hogares y uso de las remesas internas e internacionales en México. Desarrollo y Sociedad, 113-141. Obtenido de http://www.scielo.org.co/pdf/dys/n78/ n78a04.pdf

Pérez, M. A. (2013). ¿Migración determinada por el pais de Origen o de Destino? Dinero, 2.

Pinzón, J. E., \& Silva Arias, A. C. (2016). Incidencia de las remesas en la condición de pobreza de los hogares Colombianos (2015). Revista de estudiantes facultad de ciencias económicas.

Reyes, R., \& Gijon, A. (julio de 2005). El mito de 


\section{USO Y DEPENDENCIA DE LAS REMESAS EXTERIOR EN LA COMUNIDAD UNISUCREÑA}

Pérez, M. A. (2013). ¿Migración determinada por el pais de Origen o de Destino? Dinero, 2.

Pinzón, J. E., \& Silva Arias, A. C. (2016). Incidencia de las remesas en la condición de pobreza de los hogares Colombianos (2015). Revista de estudiantes facultad de ciencias económicas.

Reyes, R., \& Gijon, A. (julio de 2005). El mito de las remesas como fuente de desarrollo en México. México.

Rivas, A. M., \& Gonzálvez, H. (2011). El papel de las remesas económicas y sociales en las familias transnacionales colombianas. Migraciones Internacionales, 76-99.

Rivas, A. M., \& Gonzálvez, H. (2011). El papel de las remesas económicas y sociales en las familias transnacionales colombianas . Migraciones Internacionales , 76-79.

Roa Martínez, M. G. (2011). Usos de Remesas Procedentes de España en la Región Pacífica Colombiana. CS, 45-80.

Roa, M. (Octubre de 2010). Uso e inversión de remesas procedentes de España en hogares con experiencia migratoria en Cali. Informe Final de Investigación. Cali.

Salazar, M. F. (2017). Modelo de negocios para una empresa dedicada a facilitar la adquisición de vivienda en el Eje Cafetero por parte de los colombianos residentes en Estados Unidos. Pereira, Colombia.

The World Bank. (2016). Migration and Remittances Factbook 2016. Obtenido de http:// www.worldbank.org/en/research/brief/migrationand-remittances

Uribe, J. (2005). Remesas de trabajadores y su impacto económico. Segunda Parte. Nota Editorial, Banco de la República. Obtenido de http:// www.banrep.gov.co/docum/Lectura_finanzas/pdf/ diciembre_4.pdf

Withers, M. (2017). Subdesarrollo migratorio en Sri
Lanka: crítica a las remesas como capital. Migración y Desarrollo, 71-95.

Zuñiga, N., Gil Araujo, S., Atienza Aziona, J., Malgesini, G., Niberg Sorensen, N., Nain Hear, N., . . . Herrera , G. (2005). La Migración un camino entre el desarrollo. Madrid: CIP-FUHEM. 